\title{
Comparison Between NSAID and Intra-articular Corticosteroid Injection in Frozen Shoulder of Diabetic Patients; a Randomized Clinical Trial
}

\author{
Authors \\ A. Dehghan ${ }^{1}$, N. Pishgooei ${ }^{2}$, M.-A. Salami ${ }^{3}$, S. M. M. zarch $^{3}$, R. Nafisi-moghadam ${ }^{4}$, S. Rahimpour ${ }^{2,5}$, \\ H. Soleimani ${ }^{1}$, M. B. Owlia ${ }^{6}$ \\ Affiliations \\ Affiliation addresses are listed at the end of the article
}

Key words

- frozen shoulder

- intra-articular injection

- NSAID received 13.09.2012

first decision 08.12.2012

accepted $\quad$ 02.01.2013

Bibliography

DOI http://dx.doi.org/

10.1055/s-0032-1333278

Exp Clin Endocrinol Diabetes

2013; 121: 75-79

(c) J. A. Barth Verlag in

Georg Thieme Verlag KG

Stuttgart · New York

ISSN 0947-7349

Correspondence

\section{A. Dehghan}

Assistant Professor in

Rheumatology, department of internal medicine

Shahid Sadoughi Hospital

Ibn Sina BLVD

Ghandi BLVD

Safayieh

Yazd

Iran

Tel.: + 98/8224000

Mobile phone: +98/91/2514

6500

drdehghanali@yahoo.com

adehghan@ssu.ac.ir

\section{Abstract}

$\nabla$

Introduction: Frozen shoulder or adhesive capsulitis is a relatively common encountered musculo-skeletal disease in which arouses following soft tissue involvement of glenohumeral joint and presents with pain and limitation of shoulder' active and passive motions.

The incidence of frozen shoulder among diabetic patients is about $10-20 \%$, stiffness in such patients is more severe and should be managed actively.

Local Glucocorticoid injection, NSAIDs and physiotherapy each can relief the symptoms. The aim of this study was to compare the efficacy of glenohumeral injection of Glucocorticoid with NSAIDs in frozen shoulder of diabetic patients.

Method: The randomized clinical trial study conducted during Feb 2009-Aug 2010 on diabetic patients with frozen shoulder that were referred to rheumatology and endocrinology clinics, Yazd, Iran. Diagnostic criteria of capsulitis were pain of shoulder and range of motion limitation in all directions. The patients were divided into 2

\section{Introduction \\ $\nabla$}

Adhesive capsulitis is a relatively common musculoskeletal complaint in outpatient that is due to soft tissue involvement of glenohumeral joint and is more prevalent among women more than 50 years [1,2]. Pain and restricted active and passive movement of shoulder are the most common clinical presentations [1].

Pain and stiffness of shoulder joint will appear within few months to one year; but the course in some patients may progress faster [1]. Frozen shoulder could be idiopathic or due to some predisposing factors like diabetes, inactivity, previous disorders of shoulder, cervical spondylitis, coronary artery diseases, pulmonary tuberculosis, chronic obstructive pulmonary disease groups, patients of first group received NSAID while the latter group were undergone intraarticular corticosteroid injection. After 1 week, home exercise was done for both group and evaluation of the patients after first visit was done likewise $2^{\text {nd }}, 6^{\text {th }}, 12^{\text {th }}$ and $24^{\text {th }}$ weeks. All registered data were transformed into SPSS-15 software and analyzed.

Results: Totally 57 patients (19 males (33.3\%) and 38 females (66.7\%) were included in the analysis. There was no significant difference between sex $(P=0.4)$ and age $(P=0.19)$ of patients.

No significant relation was detected between 2 groups after 24 weeks according to range of motion in flexion ( $\mathrm{P}=0.51)$, abduction $(\mathrm{P}=0.76)$, external rotation $(0.12)$ and internal rotation $(\mathrm{P}=0.91)$. Also any significant difference in pain score was not detected $(\mathrm{P}=0.91)$.

Conclusion: Based on our study, both intraarticular corticosteroid and NSAID are effective in treatment of adhesive capsulitis and there is no significant difference between efficacies of these 2 treatment modalities in diabetic patients.

(COPD), bronchial carcinoma, hyperthyroidism, hemiplegia, brain tumors, epilepsy and Parkinson disease [2,3].

Diagnosis is by history of pain and reduced range of motion and in physical examination with active and passive restricted range of motion in all directions [4]. Definitive diagnosis is made by artrography, that only amount of radiopaque solution fewer than $15 \mathrm{ML}$ can be injected into the joint [5]. Symptoms will be resolved spontaneously within 1-3 years in most of patients, but some degree of joint movement restriction will remain [5].

Prevalence of adhesive capsulitis in normal population is about $2 \%$ that in diabetic patients increases to $10-20 \%$. The prevalence is related to the duration of diabetes. The mean age of capsu- 
litis in diabetic patients is lower than general population; also duration of disease is longer and response to treatment is less. Bilateral involvement is more seen in diabetics too [6].

Capsulitis ordinary happens in $4^{\text {th }}-6^{\text {th }}$ decades of life. Disease will start silently and has 3 phases:

First phase is named 'Painful Phase' that is accompanied with pain and restricted range of motion. This phase lasts about 2-9 months. The second phase is known as 'Freezing (Adhesive) Phase' that lasts about 3-9 months. During second phase fibrosis is formed and pain decreases while range of motion is reduced more. Third phase named 'Throwing (Resolution) Phase', which pain is subsided and also the lost motions are resolved. This phase lasts about 12-18 months [5].

A number of treatments have been advocated such as rest, physiotherapy, analgesia, acupuncture, active and passive mobilisation, oral and injected corticosteroids, capsular distension, manipulation under anaesthesia and surgical capsular release. It is surprising that for such a common condition there is no consensus on the most effective treatment $[3,7]$.

Because capsulitis is one of the most prevalent complains in diabetic patients and can effect on performance and quality of life, and also because there is no definitive treatment of it, we designed this study to compare the efficacy of 2 most popular treatment modalities with together.

\section{Materials and Methods}

$\nabla$

The randomized clinical trial study was conducted on diabetic patients with adhesive shoulder capsulitis. Based on previous studies and following parameters: $\alpha=0.05, \beta=0.2$ and $d=10$, about 30 cases were needed for each group.

Data were registered in a questionnaire which was included in questions about sex, age, time of diabetes diagnosis, drug history, results of shoulder clinical examination with goniometer and pain score based on VAS (visual analogue score). Patients were chosen from all diabetic patients who were admitted to rheumatology clinic. Diagnosis was confirmed by an Internal Medicine resident with clinical examination and by detection of pain and limited range of motion in shoulder joint (Glenohumeral joint). Because there is different response to treatment between acute and chronic form of disease, patients with more than 6 months of disease were excluded from the study. Also patients with other proved causes of capsulitis like degenerative diseases, infection, fractures and stroke were excluded from the study. Other exclusion criteria's were active peptic ulcer, history of GI bleeding, history of coagulopathies and renal failure. After that screened patients were examined by a rheumatologist. To rule out other causes of disease and checking for possible confirmatory evidence of capsulitis like osteopenia, X-ray was taken for each patient. Also for diagnosis of possible secondary causes and as basic laboratory tests, CBC (Complete Blood Count), ESR (Erythrocytes Sedimentation Rate), CRP (C Reactive Protein), urea, creatinine, liver enzymes and U/A (Urine Analysis) were checked for all patients.

The researcher explained about all treatment modalities for patients and written consent was obtained from each participants. Patients were divided into 2 groups using table of random numbers. First group got NSAID as treatment while the second group underwent intra-articular injection of corticosteroids under sonography guide. Before treatment, patients were evaluated for severity of pain and extent of restricted range of motion.
Pain was assessed according to VAS (Visual Analogue Score) by using Pain Assessment Ruler (PAR). In this study pain is graded between $0-10$ levels (no pain to severe pain). Evaluation in this scaling is subjective and was done by patients and noted in questionnaire by researcher at each visit. Range of motion also was evaluated in 3 directions of flexion, abduction and external rotation by goniometer. Also internal rotation evaluated by checking the ability of patients to reach dorsum of their hand to back and checking the highest point possible on their inferior border of scapula that assessed by plus (0-4) and noted at each visit.

Considering with different response to different types of NSAIDs, $500 \mathrm{mg}$ Naproxen twice daily was administered for all patients. Anti inflammatory dose of Naproxen reaches by using 2 tablets daily and then its use is more convenient for the patient. In second group patients underwent single injection of $40 \mathrm{mg}$ triamcinolone at the start of study. The 24 gauge needle was injected between medial head of humerus and 1 centimeter' of lateral head of coracoid process and then moved directly to the posterior, superior and lateral position. To ensure the direct injection into joint capsule, injections were ultrasound-guided. After 1 week, all patients started home exercise with moving shoulder in 3 directions of flexion (maximum range of $180^{\circ}$ ), abduction (maximum range of $180^{\circ}$ ) and taking back by hand and maximum reaching digits to inferior border of scapula. This exercise recommended 3 sessions a day and 15 times at each session. Patients were evaluated at $2^{\text {nd }}, 6^{\text {th }}, 12^{\text {th }}$ and $24^{\text {th }}$ weeks. All registered data were transformed into SPSS-15 program and analyzed by repeated measures, T-test and Chi-square tests.

\section{Results}

$\nabla$

Totally 75 patients were visited. 11 patients of injection group and 7 patients of naproxen group were excluded because discontinuing the follow-up process and finally 57 patients were included in the analysis ( 28 in naproxen group and 29 in injection group), flow diagram of the study is indicated in the 8 Fig. 1. In naproxen group, 17 (60.7\%) patients were female and 11(39.3\%) patients were male while in injection group 21 (72.4\%) and 8 (27.6\%) were female and male respectively ( $\mathrm{P}=0.4$; Chi-square). Mean age of participants were $52.78 \pm 6.72$ and $55.31 \pm 7.7$ years for naproxen and injection groups respectively ( $\mathrm{P}=0.19$; T-test). Also there was no significant difference between groups according to HbA1C ( 0.25 ; Chi-square) and duration of diabetes $(\mathrm{P}=0.9$; T-test) (9.3 \pm 7 years in naproxen group vs. $9.5 \pm 5.8$ in injection group).

Mean range of motion (flexion, abduction and external rotation) were increased in fifth visit comparing with second visit significantly $(\mathrm{P}=0.001$; paired $\mathrm{T}$-test). Also mean of internal rotation improved and pain score was decreased $(\mathrm{P}=0.001$; paired T-test) ( $\bullet$ Table 1). For injection group also similar results were obtained ( $\mathrm{P}=0.001$; paired T-test) ( $\odot$ Table 2 ).

There were no significant difference between groups According to flexion ( $\mathrm{P}=0.51$; Repeated Measure), Abduction ( $\mathrm{P}=0.76$; Repeated Measure), external rotation $(P=0.12$; Repeated Measure), internal rotation and also pain score $(\mathrm{P}=0.91$ and $\mathrm{P}=0.90$ respectively; repeated measure) ( $\odot$ Table 3 ).

In comparison of mean flexion and abduction with maximum normal degree $\left(180^{\circ}\right)$, mean flexion and abduction scores at the fifth visit were closer to maximum score to some extent that had no significant relation with maximum normal range ( $\bullet$ Table 4 ). 


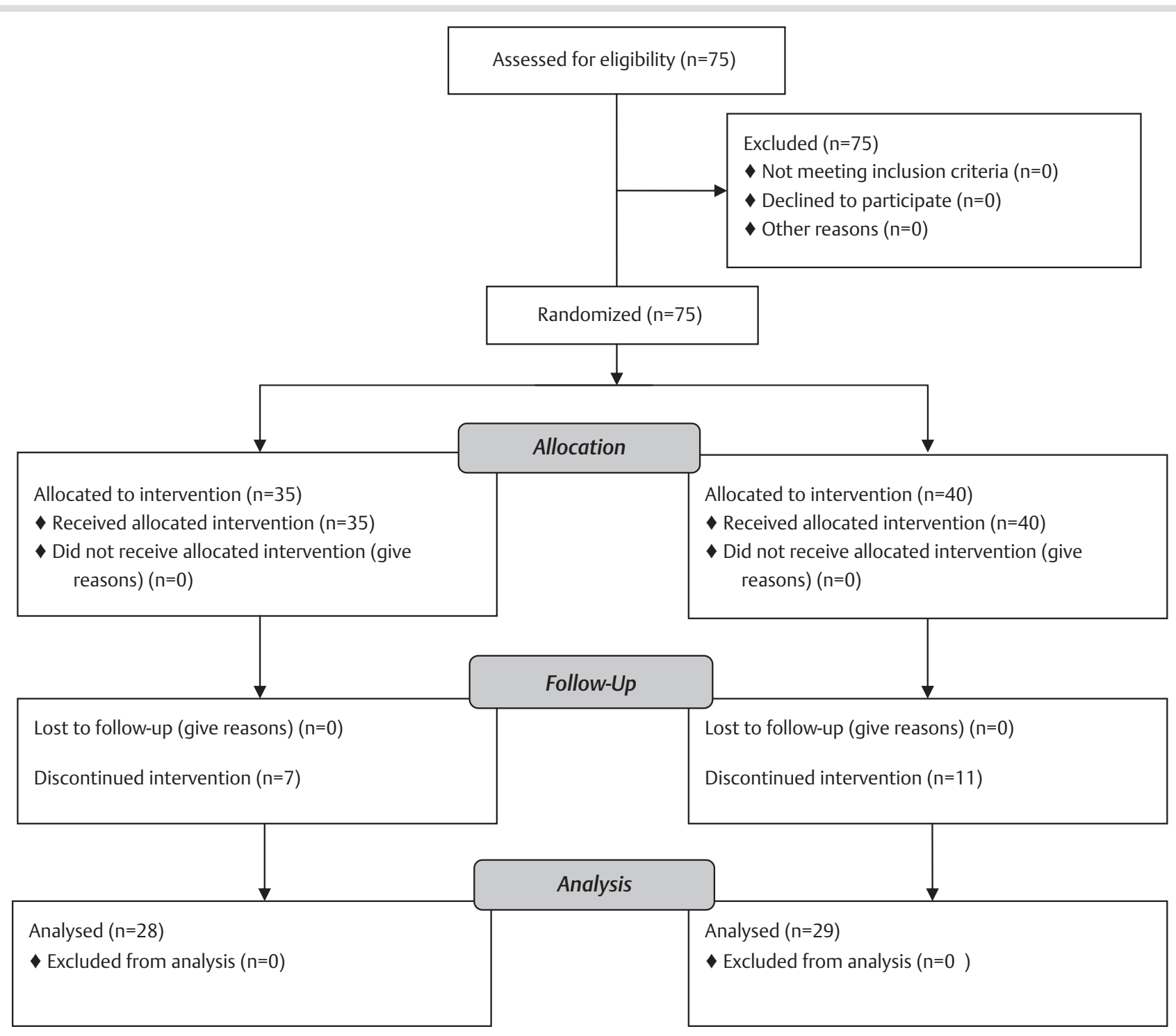

Fig. 1 Flow diagram of the study.

For naproxen group there was similar results, range of motion in abduction at fifth visit was $170^{\circ}$.

\section{Discussion}

$\nabla$

Our study compared intra-articular injection of triamcinolone with NSAID (naproxen) in diabetic frozen shoulder patients. This study was done only in diabetic patients, and adhesive capsulitis due to any other underlying causes were excluded from the study. Range of motion was detected precisely by goniometer. Also to guarantee the maximum effect of treatment, injections were done under sonography guide and also patient in NSAID group were requested to give the remnant drugs back to researcher. Patients were followed for 6 months and evaluated within 5 visits.

After 6 months of follow-up, we did not find any significant different between 2 groups according to flexion, abduction, external rotation, internal rotation and also pain score. Range of motion in patients of both groups almost returned to normal range.
There is only one study which compared intra-articular corticosteroid with oral NSAID in patients with adhesive capsulitis [8], but to our knowledge there is no study to compare these treatments in diabetic patients specifically.

Arslan et al. in 2001 [8], studied on effect of corticosteroid, physiotherapy and NSAID on adhesive capsulitis. 10 men and 10 women allocated into 2 groups. Group A underwent $40 \mathrm{mg}$ intraarticular methylprednisolone while in group B physiotherapy and NSAID was administered. Results showed that at the end of $12^{\text {th }}$ week improvement in active and passive range of motion and pain score were similar between groups. Sample size in this study was lower than ours and was not limited to diabetic patients.

In a study by Buchbinder et al., effect of oral corticosteroids was compared to placebo. They pointed out that a 3 week course of prednisolone $30 \mathrm{mg}$ daily in patients with adhesive capsulitis is better than placebo to improve pain, function, and range of motion [9].

In 2007, Russel et al. compared prednisolone and triamcinolone in painful shoulder. After 2 weeks of follow-up, improvement in pain and range of motion was seen in $92 \%$ of patients got prednisolone and $50 \%$ of patients got triamcinolone. Patients under 
treatment of prednisolone had faster recovery [10]. In these study patients had painful shoulder with any reason and duration of follow-up was only 2 weeks. In 2008 Isar Ahmad [11], compared these 2 drugs in adhesive capsulitis, but did not showed any difference between 2 groups. Also this study suggested that triamcinolone had better results in diabetic patients. This study compared diabetics and non diabetics, while our study has focused on diabetic patients only.

In 2011, Roh et al. examined the efficacy of corticosteroid injections for the treatment of adhesive capsulitis in patients with diabetes mellitus. A group of patients were undergone injection and home exercise and another group only did home exercise. In conclusion authors resulted that a corticosteroid injection in diabetic patients decreases the pain perception and accelerates the functional recovery in the early post-injection period [12].

Table 1 Mean \pm SD of variables at first and fifth visits and P-Values in Naproxen group (Paired T-test).

\begin{tabular}{|l|ccc|}
\hline Variable & Visit & Mean \pm SD & P-Value \\
\hline flexion (degree) & first visit & $107.6 \pm 15.7$ & 0.001 \\
& fifth visit & $167.6 \pm 22$ & \\
\hline abduction (degree) & first visit & $99.2 \pm 22.6$ & 0.001 \\
& fifth visit & $170 \pm 22.9$ & \\
\hline external rotation (degree) & first visit & $28.2 \pm 9.5$ & 0.001 \\
& fifth visit & $45.7 \pm 9.8$ & \\
\hline internal rotation $(+)$ & first visit & $2.6 \pm 0.87$ & 0.001 \\
& fifth visit & $0.32 \pm 0.54$ & \\
\hline pain score & first visit & $5.64 \pm 2.43$ & 0.001 \\
\hline
\end{tabular}

Table 2 Mean \pm SD of variables at first and fifth visits and P-Values in Triamcinolone group (Paired T-test).

\begin{tabular}{|llrl|}
\hline Variable & Visit & Mean \pm SD & P-Value \\
\hline flexion (degree) & first visit & $103.7 .6 \pm 22.3$ & 0.001 \\
& fifth visit & $167.4 \pm 24.2$ & \\
abduction (degree) & first visit & $90.6 \pm 21.3$ & 0.001 \\
& fifth visit & $172.9 \pm 21.6$ & \\
\hline external rotation (degree) & first visit & $30.8 \pm 11.7$ & 0.001 \\
\cline { 2 - 3 } & fifth visit & $47.4 \pm 11.4$ & \\
\hline internal rotation (+) & first visit & $2.5 \pm 1.01$ & 0.001 \\
& fifth visit & $0.24 \pm 0.43$ & \\
\hline pain score & first visit & $6.18 \pm 2.17$ & 0.001 \\
& fifth visit & $2.24 \pm 2.06$ & \\
\hline
\end{tabular}

This article did not compare the effect of corticosteroids with any oral drugs.

Smith et al. in 2005, compared intra articular injection of triamcinolone under fluoroscopy guide following with 12 sessions of physiotherapy with triamcinolone only in adhesive capsulitis patients. Results showed that corticosteroid with physiotherapy have better results than using only corticosteroids [13].

Widiastuti-Samekto et al. in 2010 compared injection of corticosteroid with oral corticosteroid in 26 patients with adhesive capsulitis. Based on results cure rate of injection group was 5.8 times more than oral group and after a week $62 \%$ of patients in injection group remitted while only $14 \%$ in oral group had remission. Compared to our study, this study had smaller sample size and follow-up period was shorter than our study too [14]. Sakeni et al. in Turkey surveyed on effect of corticosteroid injection accompanied with exercise at home. Based on this study intra-articular corticosteroid has additive effect to exercise in acceleration of remission especially during first week. There was no comparison in this study with other treatment modality [15]. A systematic review was done by Bruce Arroll [16] to determine improvement of symptoms of intra-articular and subacromial injections of corticosteroid for rotator cuff tendonitis and frozen shoulder. 7 articles that compared corticosteroid vs. placebo and 3 articles compared corticosteroid vs. NSAID were included in the study. The results indicated that sub acromial injection of corticosteroid is suitable for improvement of tendonitis and possibly is more appropriated than NSAID. But there is lack of evidence for determination of intra-articular injection of corticosteroids in adhesive capsulitis.

A valuable Meta analysis in 2012 by Maund et al., resulted that there may be benefit from adding a single intra-articular steroid injection to home exercise in patients with frozen shoulder of less than 6 months duration. This study also reported contradictory results from some other studies in this context and concluded that there is limited clinical evidence on the effectiveness of treatments for primary frozen shoulder [17].

In our study we didn't find any difference between intra-articular injection and NSAID. Because diabetic patients had simultaneous complications such as nephropathy or hypertension and considering with potential side effects of NSAIDs like gastrointestinal bleeding and also the use of aspirin at same time by many diabetic patients that can intensify the side effects of NSAIDs, it seems that administration of 1 injection of triamcinolone had equal treatment effects with less side effects and can be suggested as the method of choice in diabetic patients.

Table 3 Mean \pm SD of variables at different visits in 2 groups (repeated measures).

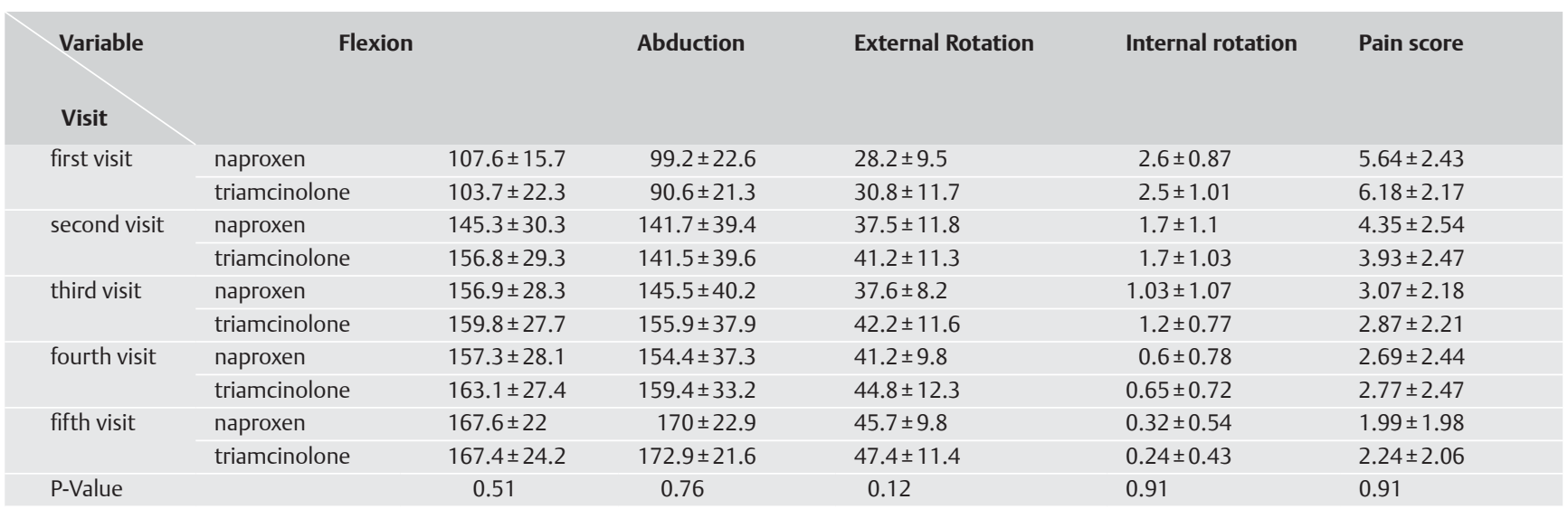


Table 4 Comparison of flexion and abduction mean \pm SD values to maximum normal values $\left(180^{\circ}\right)$ (one sample T-test).

\begin{tabular}{|lllll|}
\hline Group & Variable & Mean & SD & P-Value \\
\hline naproxen & flexion 3 & 156.9 & 28.3 & 0.001 \\
\hline triamcinolone & & 159.8 & 27.7 & 0.01 \\
\hline naproxen & flexion 4 & 157.3 & 28.1 & 0.001 \\
\hline triamcinolone & & 163.1 & 27.4 & 0.03 \\
\hline naproxen & flexion 5 & 167.6 & 22 & 0.06 \\
\hline triamcinolone & & 167.4 & 24.2 & 0.09 \\
\hline naproxen & abduction 3 & 145.5 & 40.2 & 0.001 \\
\hline triamcinolone & & 155.9 & 37.9 & 0.02 \\
\hline naproxen & abduction 4 & 154.4 & 37.3 & 0.01 \\
\hline triamcinolone & & 159.4 & 33.2 & 0.02 \\
\hline naproxen & abduction 5 & 170 & 22.9 & 0.29 \\
\hline triamcinolone & & 172.9 & 21.6 & 0.90 \\
\hline
\end{tabular}

\section{Conclusion}

Based on our study, intra articular corticosteroid and NSAID are effective in treatment of adhesive capsulitis both and there is no significant difference between these 2 treatment modalities in diabetic patients. Based on our knowledge our study was the first study on comparison of corticosteroid and NSAID in frozen shoulder of diabetic patients. Because diabetic patients have other condition such as hypertension or nephropathy, 1 intraarticular injection may be more appropriated in comparison to receiving NSAID for 1 month.

Conflict of interest: This study was under financial support of faculty of medicine, Shahid sadoughi University of medical sciences, Yazd, Iran as part of Dr. Nafiseh Pishgooie dissertation to be graduated as internist. Other authors declare no conflict of interest.

\footnotetext{
Affiliations

${ }^{1}$ Assistant Professor in Rheumatology, department of internal medicine Shahid Sadoughi Hospital, Shahid Sadoughi University of Medical Science, Yazd, Iran

${ }^{2}$ Resident in Internal Medicine, department of internal medicine, Shahid Sadoughi Hospital, Shahid Sadoughi University of Medical Science, Yazd, Iran

${ }^{3}$ Assistant Professor in Endocrinology, department of internal medicine, Shahid Sadoughi Hospital, Shahid Sadoughi University of Medical Science, Yazd, Iran

${ }^{4}$ Assistant Professor in Radiology, department of Radiology, Shahid Sadoughi Hospital, Shahid Sadoughi University of Medical Science, Yazd, Iran ${ }^{5}$ Internal Medicine resident, department of internal medicine, Shahid Sadoughi Hospital, Shahid Sadoughi University of Medical Science, Yazd, Iran

${ }^{6}$ Associate Professor in Rheumatology, department of internal medicine, Shahid Sadoughi Hospital, Shahid Sadoughi University of Medical Science, Yazd, Iran
}

\section{References}

1 Frieman BG, Albert TJ, Fenlin JM Jr. Rotator cuff disease: A review of diagnosis, pathophysiology and current trends in treatment. Arch Phys Med Rehabil May 1994; 75: 604-609

2 Bjelle A. Epidemiology of Shoulder Problems. Baillieres Clin Rheumatol Dec 1989; 3: 437-451

3 Palmer KT, Cooperc Walker-Bone $K$ et al. Use of Keyboards and Symptoms in the Neck and Arm: Evidence From a National Survey. Occup Med 2001; 51: 392-395

4 de Jong BA, Dahmen $R$, Hogeweg JA et al. Intra-Articular Triamcinolone acetonide Injection in Patients with Capsulits of the Shoulder: A comparative Study of Two dose regimes. Clin Rehabil 1998; 12: 211-215

5 Macforlane G. Upper Limb Disorders. In: Silman A, Hochberg MC. (eds.). Epidemiology of the rheumatic disease. Oxford Medical Publications, 2001; 339-366

6 Ryans I, Montgomery A, Galway R et al. Randomized Controlled Trial of Intra-Articular Triamcinolone and/or Physiotherapy in shoulder Capsulits. Rheumatology (OXFORD) 2005; 44: 522-535

7 Sharma S. Management of frozen shoulder - conservative vs surgical? Ann R Coll Surg Engl Jul 2011; 93: 343-344 discussion 345-346

8 Arslan S, Celiker R. Comparison of the Efficiency of Local Corticosteroid Injection and Physical Therapy for the Treatment of Adhesive Capsulits. Rheumatol Int 2001; 21: 20-23

9 Buchbinder R, Hoving JL, Green $S$ et al. Short course prednisolone for adhesive capsulitis (frozen shoulder or stiff painful shoulder): a randomised, double blind, placebo controlled trial. Ann Rheum Dis 2004; 63: 1460-1469

10 Russell J. Shoulder Adhesive Capsulits: Systematic Review of Randomized Trials Using Multiple Corticosteroid Injections. British Journal of General Practice 2007; 57: 662-667

11 Isar Ahmad, Zahid Askar. Intra-articular injection of Methylprednisolone for Idiopathic frozen shoulder. J Med Sci (Pishawar print) 2009; 17: 16-18

12 Roh YH, Yi SR, Noh JH et al. Intra-articular corticosteroid injection in diabetic patients with adhesive capsulitis: a randomized controlled trial. Knee Surg Sports Traumatol Arthrosc Nov 242011

13 Smith F. American College of Rheumatology Intera-Articular Corticosteroids, Supervised Physiotherapy, Adhesive Capsulitis of the Shoulder. Arthritis \& Rheumatism 2005; 48: 829-838

14 Widiastuti-Samekto M, Sianturi GP. Comparison of oral Route corticosteroid and intra-articular corticosteroid injection. Med J Malaysia 2004; 59: 312-316

15 Sakeni RA, Al-Nimer MS. Comparison between intraarticular triamcinolone acetonide and methylprednisolone acetate injections in treatment of frozen shoulder. Saudi Med J 2007; 28: 707-712

16 Arroll B, Goodyear-Smith F. Corticosteroid Injections of Painful Shoulder: A Meta-Analysis. Br J Gen Pract 2005; 55: 224-228

17 Maund E, Craig D, Suekarran S et al. Management of frozen shoulder: a systematic review and cost-effectiveness analysis. Health Technol Assess 2012; 16: 1-264 